\title{
Caractérisation de l'alimentation des jeunes enfants âgés de 6 à 36 mois en milieu rural et urbain du Sud- Bénin
}

\author{
Sandrine E. Kouton¹, Waliou Amoussa Hounkpatin², Vénérande Y. Ballogou¹, Jaurès H. Lokonon², \\ Mohamed M. Soumanou ${ }^{1}$ \\ 1 Unité de Recherche en Génie Enzymatique et Alimentaire/École Polytechnique d'Abomey Calavi (EPAC)/ Université \\ d'Abomey-Calavi (UAC), 01 BP 2009 Cotonou, Bénin \\ 2Département de Nutrition et Sciences Alimentaires/Faculté des Sciences Agronomiques (FSA)/ Université \\ d'Abomey-Calavi (UAC) 01 BP 526 Cotonou, Bénin \\ *Auteur correspondant : msoumanoufr@yahoo.fr Tél : 00 (229) 97877870
}

Original submitted in on $16^{\mathrm{h}}$ November 2016. Published online at www.m.elewa.orgon $28^{\text {th }}$ February 2017 http://dx.doi.org/10.4314/iab.v110i1.13

\begin{abstract}
RESUME
Objectif : La période d'alimentation complémentaire est une période critique pour la croissance de l'enfant. Cette étude vise à décrire l'alimentation des jeunes enfants âgés de 6 à 36 mois en milieu rural et urbain du Sud-Bénin.

Méthodologie et Résultats : Une enquête transversale a été menée sur 600 mères ayant des enfants âgés de 6 à 36 mois dans les zones rurales et urbaines du sud-Bénin, où la prévalence de la malnutrition chronique est élevée (prévalence variant de $40 \%$ à $43 \%$ ). Les caractéristiques socio-économiques de la population, les connaissances (définition correcte de l'alimentation complémentaire) et les pratiques (âge d'introduction de l'aliment de complément, les caractéristiques des aliments de complément) ont été documentées. L'analyse en composante principale a été utilisée pour identifier les associations entre les caractéristiques socioéconomiques de la population et les pratiques d'alimentation des jeunes enfants de 6 à 36 mois. Dans l'ensemble, $65 \%$ des mères méconnaissent la notion d'alimentation complémentaire. L'introduction précoce des aliments de complément est observée dans $48.5 \%$ des cas. La bouillie dans $99.16 \%$ des cas est le premier aliment de complément donné aux enfants. Globalement, $67 \%$ des mères utilisent des bouillies locales, peu enrichies avec des matières protéiques. La consommation de bouillie issue des farines infantiles vendues dans le commerce est associée au milieu urbain alors que la bouillie locale est associée au milieu rural.

Conclusion et application des résultats : Les bouillies locales sont peu enrichies avec des matières protéiques et les fréquences de consommation journalière sont faibles. Ces résultats révèlent l'importance d'améliorer les procédés et formulations traditionnelles des bouillies, stratégies endogènes durables qui méritent d'être encouragées par l'éducation nutritionnelle pour la production de bouillies enrichies à haute valeur nutritionnelle avec des matières premières locales accessibles pour couvrir les besoins nutritionnels et énergétiques des jeunes enfants, gage d'une croissance optimale.
\end{abstract}

Mots clés : Alimentation, enfants de 6 à 36 mois, bouillies, Bénin. 
Characterization of the feeding of young children aged 6 to 36 months in rural and urban areas of the South Benin

\section{ABSTRACT}

Objective : The period of complementary feeding is a critical period for the growth of the child. This study aims to describe the feeding of young children aged 6 to 36 months in rural and urban areas of the South Benin. Methodology and Results : A cross sectional survey was conducted on 600 mothers having children aged 6 to 36 months in rural and urban areas of the South Benin, where the prevalence of chronic malnutrition is high (prevalence between $40 \%$ to $43 \%$ ). The socio economics data, knowledge (correct definition of complementary feeding) and practices (age of introduction of Complementary Foods, characteristics of Complementary Foods) were collected. Analysis of principal component was used to identify the associations between the socio economics data and feeding practices for 6 to 36 months' young children. About, $65 \%$ of mothers ignore the concept of complementary feeding. The early introduction of complementary foods was observed in $48.5 \%$ of cases. Porridge in $99.16 \%$ of cases is the first complementary food given to children. Overall, $67 \%$ of mothers use local porridges slightly enriched with protein feeds. Porridge based on commercial infant flour consumption is associated with the urban area while local porridge is associated with rural areas.

Conclusion and application and results: Local porridge is poorly enriched with protein feeds in rural areas and the frequencies of daily consumption are low. These results reveal the importance of improving the processes and traditional formulations of local porridges. Sustainable strategies that need to be encouraged by mothers 'nutritional education for the production of enriched porridges with high nutritional value and local raw materials accessible in order to cover the nutritional requirements and energy need of young children, pledge of an optimal growth.

Key words: feeding, children of 6 to 36 months, porridges, Benin.

\section{INTRODUCTION}

L'OMS recommande qu'à partir de six mois, âge auquel l'allaitement maternel seul ne suffit plus pour garantir une meilleure croissance des enfants et une santé optimale, soient introduits des aliments de complément adaptés (OMS, 2003). Le nourrisson est particulièrement vulnérable pendant la période de transition où commence l'alimentation complémentaire (OMS, 2012). Cette période constitue une phase à haut risque dans la vie des nourrissons, car mal conduit, l'alimentation complémentaire hisse la malnutrition pluricarentielle au rang des problèmes cruciaux de santé chez les nourrissons (Azagoh-K. et al., 2013 ; Victora et al., 2016). La malnutrition touche les enfants de moins de cinq ans principalement dans la période d'alimentation complémentaire dans le monde en général et dans les pays en développement en particulier et constitue la cause de la moitié au moins des décès d'enfants (Black et al., 2013; Dolan et al., 2015). Les premiers aliments de complément donnés aux enfants africains sont des bouillies à base de céréales peu enrichies par des légumineuses locales (pâte d'arachide ou farine torrifiée de soja), du lait ou de la poudre de poisson séché (Trèche, 2002 ; Traore et al., 2005). En réalité, les aliments de complément sont définis comme les premiers aliments (liquides ou solides) introduits dans l'alimentation du bébé en addition au lait maternel (Brown \& Lutter, 2000). Au Bénin, les aliments de complément sont constitués essentiellement de bouillies à base de farine de maïs, mil ou sorgho (46\%) et des mélanges ou recettes souvent mal faits de céréales et de légumineuses (42\%) (Atègbo, 1993, Carbonel, 1996). Les bouillies traditionnelles à base de maïs données aux enfants ont une densité énergétique très faible qui est de l'ordre de 36 à $60 \mathrm{cal} / 100 \mathrm{ml}$ pour les bouillies à base de mil et sorgho au Burkina Faso, 44 à $64 \mathrm{cal} / 100 \mathrm{ml}$ au Gabon et $60 \mathrm{cal} / 100 \mathrm{ml}$ au Congo (Salle, 2009). Les densités en micronutriments sont également faibles. Au Bénin, les pratiques d'alimentation complémentaire ne sont pas optimales. En effet, $73 \%$ des nourrissons de 6-8 mois reçoivent des aliments solides, semi-solides ou mous. Bien que plus de la moitié des enfants 6-23 mois $(52,5 \%)$ ont une fréquence minimum de repas, 
seulement $18.5 \%$ ont reçu au moins 2 repas lactés durant le jour précédent l'enquête (INSAE, 2015). Les aliments de complément introduits par les mères sont pour la plupart des bouillies simples de maïs et/ou de sorgho fermenté ou non, des bouillies obtenues avec des pâtes de maïs recyclées. Les bouillies sont peu enrichies avec des matières protéiques et la fréquence de consommation journalière est faible. Les enfants sont habitués à consommer des plats à base de matière glucidique exclusivement comme la pâte de mais accompagnée d'une sauce ou du piment écrasé sans viandes ni poisson (UNICEF, 2014). Par ces pratiques, l'utilisation d'aliments de complément de qualité médiocre et la conduite inadéquate du processus d'alimentation complémentaire, expliqueraient en partie la prévalence de $34 \%$ du retard de croissance observées chez les enfants béninois en âge préscolaire (INSAE, 2015). Les conséquences économiques de la malnutrition chronique représentent des pertes de 11 pourcent du produit intérieur brut (PIB) chaque année en Afrique et en Asie, alors que la prévention de cette malnutrition offre $16 \$$ des rendements sur investissement pour chaque 1 \$ dépensé (de Onis et al., 2013 ; GNR, 2016). L'adéquation de l'alimentation complémentaire et l'alimentation des jeunes enfants en général, dépend de nombreux facteurs notamment des facteurs liés à l'aliment (composition nutritionnelle, densité énergétique, caractéristiques organoleptiques), ceux liés à l'enfant (état de santé, sexe, capacités gastriques) puis à la personne qui

\section{METHODOLOGIE}

Zone d'étude et échantillonnage : Cette étude a été menée en milieu urbain et rural dans quatre départements du Sud-Bénin où les prévalences de la malnutrition chronique dépassent le seuil critique (prévalence variant de $40 \%$ à $43 \%$ ). Une commune a été choisie dans chaque département. Les communes concernées en milieu rural sont celles d'Adja-Ouèrè, de Dogbo et en milieu urbain, celles de Porto-Novo et Cotonou. Six cent (600) mères d'enfants âgés de 6 à 36 mois ont été enrôlées au cours de l'enquête, à raison de cent-cinquante couples (150) mère-enfant par département. Le choix des quartiers ou villages enquêtés a été fait de manière aléatoire grâce à la table des nombres aléatoires par numérotation et tirage au sort. La s'occupe de l'enfant (Trèche, 2002 ; Forouzanfar et al., 2015). Les connaissances inadéquates concernant les aliments de complément et les pratiques d'alimentation complémentaire inappropriées des jeunes enfants affectent plus les enfants que le manque de nourriture dans la malnutrition (Amoussa-Hounkpatin, 2011 ; Zannoutchoco et al., 2011). Dans ces conditions, l'alimentation complémentaire telle que conduite actuellement par les mères des milieux ruraux surtout du Sud-Bénin ne pourra pas combler les besoins nutritionnels et énergétiques des nourrissons et les exposent à différentes formes de malnutrition qui hypothèquent leur croissance voire leur survie. Dans le processus de mise en route des mesures adéquates d'amélioration des différents procédés de formulation des farines infantiles dans les milieux ruraux en vue de promouvoir de bonnes pratiques d'alimentation des jeunes enfants, il est nécessaire de comprendre d'abord les fondements sociaux et économiques actuels qui déterminent l'alimentation complémentaire introduite par les mères ou gardiennes d'enfants. La présente étude se propose de décrire les connaissances, pratiques d'alimentation du jeune enfant et les bouillies introduites comme aliment de complément des enfants de 6 à 23 mois et aussi comme aliment fréquemment consommé par les jeunes enfants de plus de 23 mois. Aussi, cette étude se propose de rechercher les facteurs socio-économiques associés à l'alimentation des jeunes enfants en zone urbaine et rurale au Sud-Bénin.

méthode de la bouteille vide a été utilisée pour le choix des ménages. Dans chaque ménage, un enfant est enquêté. Les critères d'éligibilité des enfants retenus dans l'échantillon ont été d'appartenir à la classe d'âge de 6 à 36 mois au cours de l'enquête et de recevoir d'autres types d'aliments en dehors du lait maternel.

Outils de collecte et considérations éthiques : Les données ont été recueillies à domicile au moyen d'un questionnaire comportant deux parties notamment les caractéristiques socio-économiques de la population et les connaissances et pratiques d'alimentation des jeunes enfants. Après vérification de l'éligibilité de l'enfant, un entretien dans le ménage sélectionné est fait afin de l'informer plus précisément des objectifs de l'étude pour 
susciter l'adhésion du ménage. En cas d'accord, le consentement matérialisé est recueilli sous forme de signature du chef de ménage apposée sur la fiche de consentement éclairé.

Analyses statistiques: Les données ont été saisies sous Excel 2013. Les différences significatives entre les pourcentages de facteurs ont été recherchées grâce au test de chi². Selon la distribution des fréquences dans la population d'étude, l'âge d'introduction des aliments de compléments a été regroupé en deux catégories (âge d'introduction $\leq 3$ mois et âge d'introduction $>3$ mois). II en est de même s'agissant de la fréquence de

\section{RESULTATS}

Caractéristique de la population: L'âge moyen des mères était de $28,44 \pm 4,34$ ans avec des extrêmes allant de 18 à 41 ans. Près de $43,66 \%$ des mères étaient sans activité génératrice de revenu, $24 \%$ étaient primipares, $48,33 \%$ avaient un niveau socio-économique bas, $60,66 \%$ étaient issues d'un milieu rural et 30\% étaient analphabètes. Les moyens d'information étaient la télévision $(39 \%$ ) et la radio $(61 \%)$. En milieu rural, $59 \%$ de mères étaient analphabètes, $67,33 \%$ étaient sans activité génératrice de revenu, $90 \%$ des ménages avaient un niveau socio-économique bas alors qu'en milieu urbain $51 \%$ des mères avaient un niveau supérieur, $80 \%$ avaient une activité génératrice de revenu, 67,33\% avaient un niveau socio-économique élevé (Tableau 1). Connaissances et pratiques des mères relatives à l'alimentation complémentaire : Dans l'ensemble, $35 \%$ consommation journalière des bouillies (en 1 à 2 fois et supérieure ou égale à 3 fois par jour). Des analyses statistiques bivariées ont été réalisées avec le logiciel Statistica (Program for Social Sciences, version 11). L'association entre l'alimentation des jeunes enfants et les caractéristiques socio-économiques des ménages a été appréciée au moyen d'une analyse en composante principale avec logiciel Minitab (version $n^{\circ} 6$ ). Lorsque $p$ value est $<0,05$, les différences sont significatives et lorsque $p$-value $<0,001$, elles sont hautement significatives.

des mères ont défini correctement l'alimentation complémentaire. En milieu rural et urbain respectivement $5 \%$ et $65 \%$ de mères ont pu définir correctement l'alimentation complémentaire. En ce qui concerne l'âge d'introduction d'aliments de complément, $50.5 \%$ de mères ont déclaré que les aliments de complément peuvent être introduits à six (06) mois. Une différence significative $(p=$ 0.0001 ) a été observée entre les déclarations des mères selon qu'elles soient en milieu rural ou urbain. En milieu rural, $74 \%$ de mères ont indiqué que les aliments de complément peuvent être introduits avant six (06) mois alors qu'en milieu urbain cette proportion n'est que de $10 \%$. En milieu urbain, $85 \%$ de mères ont indiqué que les aliments de compléments peuvent être introduits à six (06) mois contre $16 \%$ en milieu rural (Tableau 2).

Tableau 1 : Caractéristiques de la population

\begin{tabular}{|c|c|c|c|c|c|c|}
\hline \multirow[t]{2}{*}{ Caractéristiques } & \multicolumn{2}{|c|}{ Milieu rural } & \multicolumn{2}{|c|}{ Milieu urbain } & \multicolumn{2}{|c|}{ Ensemble } \\
\hline & $\mathrm{n}$ & $\%$ & $n$ & $\%$ & $\mathrm{n}$ & $\%$ \\
\hline \multicolumn{7}{|c|}{ Age des mères (ans) } \\
\hline$<25$ & 60 & 20 & 15 & 05 & 75 & 12,5 \\
\hline $25-35$ & 236 & 78,66 & 277 & 92,33 & 513 & 85,5 \\
\hline$>35$ & 04 & 1,33 & 08 & 2,66 & 12 & 02 \\
\hline \multicolumn{7}{|c|}{ Niveau d'instruction } \\
\hline Jamais scolarisée & 177 & 59 & 06 & 02 & 183 & 30 \\
\hline Primaire & 85 & 28,33 & 67 & 22,33 & 152 & 26 \\
\hline Secondaire & 33 & 11 & 74 & 24,66 & 107 & 18 \\
\hline Supérieur & 05 & 1,66 & 153 & 51 & 158 & 26 \\
\hline \multicolumn{7}{|c|}{ Activité génératrice de revenu } \\
\hline Sans & 202 & 67,33 & 60 & 20 & 262 & 43,66 \\
\hline Avec & 98 & 32,66 & 240 & 80 & 338 & 56,34 \\
\hline \multicolumn{7}{|l|}{ Parité } \\
\hline 1 enfant & 40 & 13,33 & 105 & 35 & 145 & 24 \\
\hline 2-3 enfants & 200 & 66,66 & 170 & 56,66 & 370 & 62 \\
\hline 4 et plus & 60 & 20 & 25 & 08,33 & 85 & 14 \\
\hline
\end{tabular}



en milieu rural et urbain du Sud -Bénin

\begin{tabular}{l|c|c|c|c|c|c}
\hline $\begin{array}{l}\text { Origine } \\
\text { Milieu rural }\end{array}$ & 275 & 91,66 & 89 & 29,66 & 364 & 60,66 \\
$\begin{array}{l}\text { Milieu urbain } \\
\text { Niveau socio-économique du ménage }\end{array}$ & 08,33 & 211 & 70,33 & 236 & 39,34 \\
\begin{tabular}{l|c|c|c|c|c|c} 
Bas \\
Moyen
\end{tabular} & 270 & 90 & 20 & 6,66 & 290 & 48,33 \\
$\begin{array}{l}\text { Élevé } \\
\text { Moyens d'information du ménage }\end{array}$ & 14 & 04,66 & 78 & 26 & 92 & 15,33 \\
$\begin{array}{l}\text { Télévision } \\
\text { Radio }\end{array}$ & 05,33 & 202 & 67,33 & 218 & 36,34 \\
\hline
\end{tabular}

$\mathbf{n}$ : Effectif et (\%) : Pourcentages de mères dans la catégorie

Pratique des mères relatives à l'alimentation complémentaire : L'introduction précoce des aliments de complément a été déclarée dans $48,5 \%$ des ménages (Tableau 3). II existe une différence significative entre l'âge d'introduction déclaré des aliments de complément et les milieux ( $p=0.0001$ ). En milieu rural, $64 \%$ de mères ont déclaré introduire les aliments de complément en temps opportun tandis que $73,33 \%$ de mères en milieu urbain ont déclaré introduire les aliments de complément avant six (06) mois.

Tableau 2: Connaissances des mères relatives à l'âge d'introduction des aliments de complément (AIAC)

\begin{tabular}{l|c|c|c|c|c|c}
\hline \multirow{2}{*}{ Réponses des mères } & \multicolumn{2}{|c|}{ Milieu rural } & \multicolumn{2}{c|}{ Milieu urbain } & \multicolumn{2}{c}{ Ensemble } \\
\cline { 2 - 7 } Inférieur à six mois & $\mathbf{n}$ & $\%$ & $\mathbf{n}$ & $\%$ & $\mathbf{n}$ & $\%$ \\
A partir de six mois & 222 & 74 & 30 & 10 & 252 & 42 \\
Après six mois & 48 & 16 & 255 & 85 & 303 & 50,5 \\
\hline
\end{tabular}

$\mathbf{n}$ : Effectif et (\%) : Pourcentages de mères dans la catégorie

Raison de précocité d'introduction d'aliment de complément: Les mères enquêtées affirment être sujettes aux situations (manque de lait, survenue de grossesse, reprise de travail et les prises de médicaments) qui les obligent à introduire avant six (06) mois les aliments de complément. Les principales causes évoquées par les mères enquêtées étaient la reprise de travail (42\%), évoqué surtout en milieu urbain et le manque de lait $(42 \%)$. La reprise de travail des mères reste en milieu urbain, la première contrainte $(80 \%)$ d'introduction précoce d'aliment de complément tandis qu'en milieu rural une survenue de grossesse (56\%) constitue la première contrainte (Tableau 4).

Tableau 3 : Déclarations des mères relatives à l'introduction des aliments de complément

\begin{tabular}{l|c|c|c|c|c|c}
\hline & \multicolumn{2}{|c|}{ Milieu rural } & \multicolumn{2}{c|}{ Milieu urbain } & \multicolumn{2}{c}{ Ensemble } \\
\cline { 2 - 7 } Déclarations des mères & $\mathbf{n}$ & $\%$ & $\mathbf{n}$ & $\%$ & $\mathbf{n}$ & $\%$ \\
Introduction précoce (IAP) & 71 & 23,66 & 220 & 73,33 & 291 & 48,5 \\
Introduction en temps & 192 & 64 & 65 & 21,66 & 257 & 42,84 \\
opportun (IATO) & & & & & & \\
Introduction tardive (IAT) & 37 & 12,33 & 15 & 05 & 52 & 08,66 \\
\hline
\end{tabular}

$\mathbf{n}$ : Effectif et (\%) : Pourcentages de mères dans la catégorie

Types de bouillies proposées aux enfants : La bouillie dans $99,16 \%$ des cas est le premier aliment de complément associé au lait maternel. En milieu rural et urbain, respectivement $6 \%$ et $77,50 \%$ de mères utilisent des bouillies issues de farines infantiles vendues dans le commerce. Dans l'ensemble, $67 \%$ des mères utilisent des bouillies locales. En milieu rural et urbain, $94 \%$ et $40 \%$ de mères respectivement utilisent des bouillies locales.
Concernant l'enrichissement des bouillies avec des matières protéiques, $55 \%$ des bouillies sont enrichies et respectivement $18 \%$ et $88,33 \%$ de bouillies sont enrichies en milieu rural et urbain. Une différence hautement significative a été observée entre les fréquences d'utilisation de bouillies locales en milieu rural et urbain et les prévalences de bouillies enrichies dans les milieux rural et urbain $(p=0.000)$. 
Kouton et al., J. Appl. Biosci. 2017 Caractérisation de l'alimentation des jeunes enfants âgés de 6 à 36 mois en milieu rural et urbain du Sud -Bénin

Tableau 4 : Principales raisons évoquées pour une introduction précoce d'aliment de complément

\begin{tabular}{l|c|c|c|c|c|c}
\hline & \multicolumn{2}{|c|}{ Milieu rural } & \multicolumn{2}{c|}{ Milieu urbain } & \multicolumn{2}{c}{ Ensemble } \\
\hline & $\mathbf{n}$ & $\%$ & $\mathbf{n}$ & $\%$ & $\mathbf{n}$ & $\%$ \\
\hline Raisons évoquées & 129 & 43 & 123 & 41 & 252 & 42 \\
Manque de lait & 12 & 4 & 30 & 10 & 42 & 07 \\
Refus d'allaiter au sein & 12 & 4 & 240 & 80 & 252 & 42 \\
Reprise du travail & 30 & 10 & 24 & 8 & 54 & 09 \\
Survenue de complications & 51 & 17 & 165 & 55 & 216 & 36 \\
L'enfant ne croît pas bien & 0 & 0 & 15 & 5 & 15 & 2,5 \\
Raisons esthétiques & 0 & 0 & 0 & 0 & 0 & 0 \\
A la demande du mari & 168 & 56 & 12 & 4 & 180 & 30 \\
Survenue de grossesse & 09 & 3 & 45 & 15 & 54 & 09 \\
Prise de médicaments & \multicolumn{2}{|c|}{} &
\end{tabular}

$\mathbf{n}$ : Effectif et (\%) : Pourcentages de mères dans la catégorie

Caractéristiques des bouillies locales: Les bouillies locales sont élaborées avec des céréales comme le maïs, le sorgho et le mil. Des matières protéiques comme le soja sont peu utilisées. Le maïs est l'aliment de base le plus fréquemment utilisé pour la préparation des bouillies locales. Les bouillies sont élaborées avec des pâtes de maïs fermentées présentées sous plusieurs formes comme le "ogui "obtenu par trempage et fermentation des grains de maïs" et le "gbahoungba" obtenu par fermentation de la farine de maïs diluée dans de l'eau". En milieu rural, $64 \%$ et $6 \%$ de bouillies sont préparées respectivement à base de maïs et de sorgho. Le sorgho est peu utilisé et représente la seconde céréale utilisée par les mères pour la production locale des bouillies.

Fréquence de consommation journalière de bouillies : La consommation journalière de bouillie varie entre 1 et 5 fois. Dans l'ensemble, $70,66 \%$ des enfants ont reçu une fois la bouillie par jour. En milieu rural, la majorité $(70,66$ $\%$ ) des enfants ont reçu une fois la bouillie par jour et en milieu urbain la majorité des enfants $(43,33 \%)$ ont reçu trois (3) fois la bouillie par jour (Tableau 5).

Tableau 5 : Fréquence de consommation journalière des bouillies

\begin{tabular}{c|c|c|c|c|c|c}
\hline & \multicolumn{2}{|c|}{ Milieu rural } & \multicolumn{2}{c|}{ Milieu urbain } & \multicolumn{2}{c}{ Ensemble } \\
\hline Nombre de fois & $\mathbf{N}$ & $\%$ & $\mathbf{n}$ & $\%$ & $\mathbf{n}$ & $\%$ \\
\hline 1 & 212 & 70,66 & 0 & 0 & 212 & 70,66 \\
\hline 2 & 70 & 23,33 & 90 & 30 & 160 & 26,66 \\
\hline 3 & 13 & 04,33 & 130 & 43.33 & 143 & 23,83 \\
\hline 4 & 5 & 01,66 & 30 & 10 & 35 & 05,83 \\
\hline 5 & 0 & 0 & 50 & 16.66 & 50 & 08,33 \\
\hline
\end{tabular}

$\mathbf{n}$ : Effectif et (\%) : Pourcentages de mères dans la catégorie

Alimentation des jeunes enfants : En milieu rural, 99\% des plats étaient exclusivement à base de céréales, racines et tubercules et $40 \%$ de plats ont été accompagnés de produits carnés. Les fruits et légumes riches en vitamine A ont été consommés par 35\% des enfants et seulement $1 \%$ consomment des œufs. En revanche en milieu urbain, $99 \%$ des plats étaient exclusivement à base de céréales, racines et tubercules et $82 \%$ de plats étaient accompagnés de produits carnés. Concernant les fruits et légumes, $75 \%$ des enfants ont consommé des fruits et légumes riche en vitamine $\mathrm{A}$ (Figure 1). 


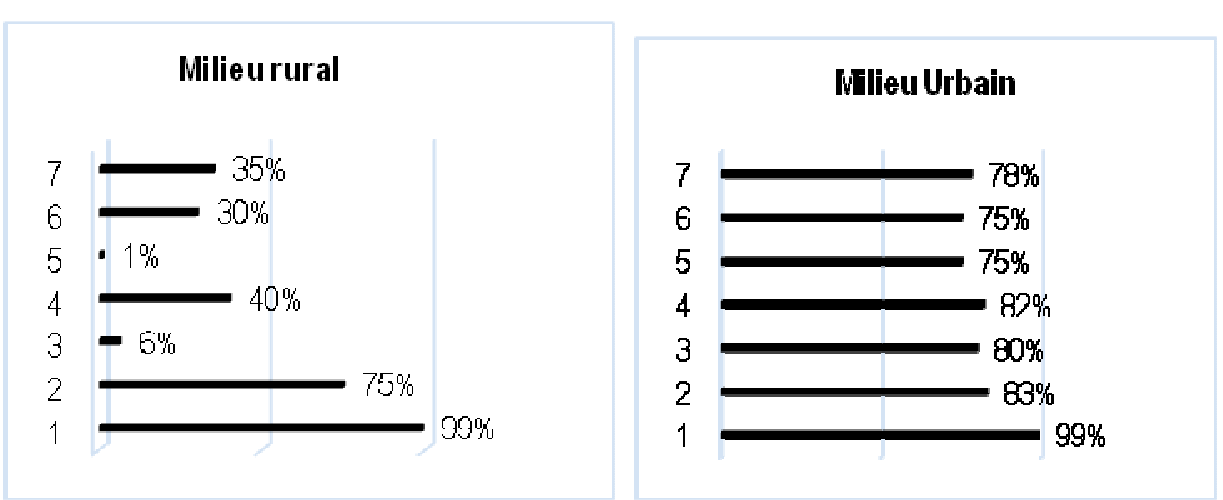

1-Céréales, racines et tubercules ; 2- Légumineuses et noix ; 3- Lait et produits laitiers ; 4-Viandes et produits carnés ; 5-CEufs ; 6Légumes et fruits riche en vitamine $A ; 7$ - Autres fruits et légumes

Figure 1 : Pourcentages des jeunes enfants ayant consommé les 7 groupes d'aliments en milieu rural et urbain

Facteurs associés à l'âge d'introduction des aliments de complément à trois mois et à la fréquence de consommation journalière de bouillie : En analyse bivariée, une association hautement significative a été observée entre l'âge d'introduction des aliments de complément et le niveau d'instruction des mères $(p<$ $0,000$; Odd Ratio $=0,10)$, l'activité des mères $(p<$ $0,014$; Odd Ratio $=0,17)$, les milieux rural et urbain $(p<$ 0,000 ; Odd Ratio $=0,30$ ) et les connaissances sur

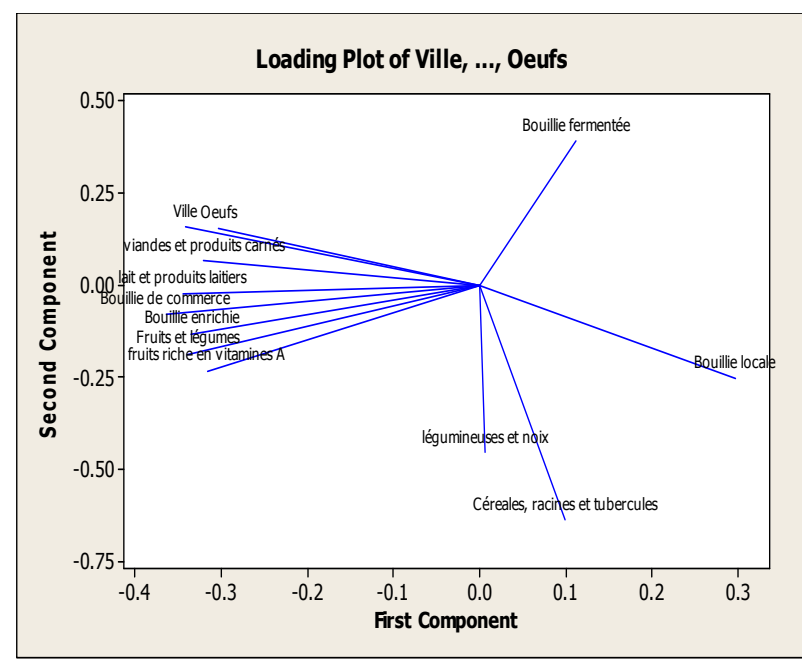

Figure 2 : Projection des variables relative aux pratiques d'alimentation des ieunes enfants dans le plan factoriel

Analyse en composante principale (ACP) des pratiques d'alimentation des jeunes enfants et des milieux : Les facteurs "Milieu", "bouillie issue des farines infantiles vendues dans le commerce", "bouillie enrichie" sont corrélés négativement sur la première composante qui regroupait $59,7 \%$ des informations initiales alors que le facteur "bouillie locale" y est corrélé positivement. II l'alimentation complémentaire $(p<0,000$; Odd Ratio $=$ 0,12 ). Les facteurs associés à la fréquence de consommation journalière de bouillie sont l'activité des mères $(p<0,000$; Odd Ratio $=2,8)$, les milieux $(p<0,000$; Odd Ratio $=3,72$ ), les niveaux socio-économiques $(p<0,019$; Odd Ratio $=3,63)$ et les connaissances relatives à l'alimentation complémentaire $(p<0,000$; Odd Ratio $=2,96$ ).

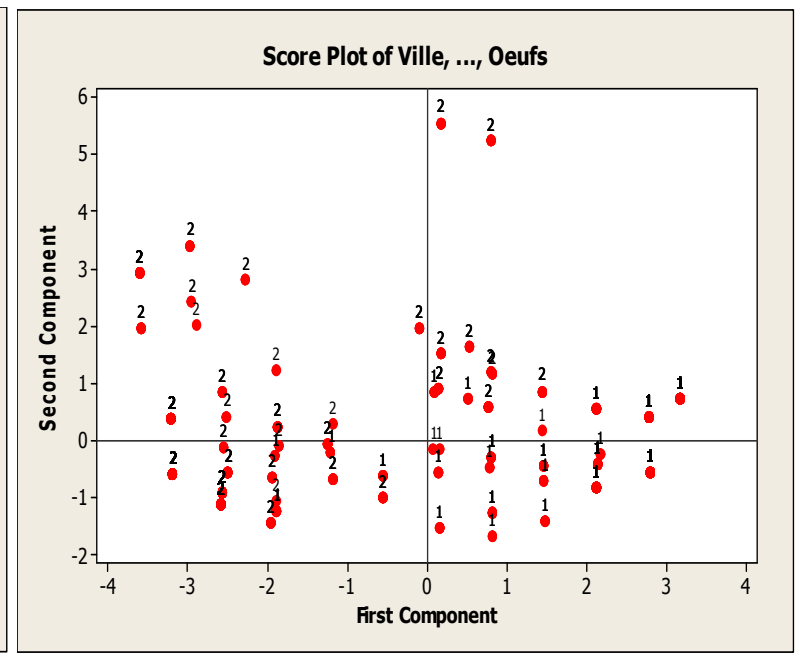

Figure 3 : Projection des milieux dans le plan factoriel suivant les pratiques d'alimentation des jeunes enfants [1-Milieu rural ; 2-Milieu urbain

ressort de la projection des milieux sur les deux premières composantes qu'il existe une association entre la bouillie issue des farines infantiles vendues dans le commerce, la bouillie enrichie et le milieu urbain alors que la bouillie locale est associée au milieu rural. La même analyse appliquée à l'alimentation des jeunes enfants et les milieux a permis de constater que la fréquence de 

en milieu rural et urbain du Sud -Bénin

consommation de céréales, racines et tubercules, de produits carnés (viandes, poissons), de fruits et légumes, de produits laitiers (lait, yaourt, fromage) et d'œufs sont

\section{DISCUSSION}

Cette étude a été conduite dans le but de décrire les pratiques d'alimentation des jeunes enfants de 6 à 36 mois. Nous avons trouvé que $65 \%$ de mères méconnaissent la notion d'alimentation complémentaire et une différence significative a été observée entre les connaissances de mères vivantes en milieux rural et urbain. Seulement, $5 \%$ des mères en milieu rural arrivent à définir correctement la notion d'alimentation de complément et $74 \%$ parmi elles déclarent que les aliments de complément peuvent être introduits avant six mois. Cette connaissance n'est pas en adéquation avec celle promue par l'OMS qui recommande que les aliments de complément ne soient introduits dans l'alimentation de jeune enfant qu'à partir de six mois (OMS, 2003). Cette proportion élevée de mères en milieu rural ayant une connaissance inadéquate sur l'âge d'introduction des aliments de complément pourrait être expliquée par un manque d'éducation nutritionnelle. II apparait clairement qu'une telle situation ne saurait aider à une bonne conduite d'alimentation complémentaire. Azagoh-K. et al. (2013) en Côte d'ivoire ont trouvé les mêmes résultats où $74 \%$ de mères méconnaissent la notion d'alimentation complémentaire. L'introduction précoce des aliments de complément a été observée dans $48,5 \%$ des cas. DiagneGueye et al. (2011) au Sénégal, ont rapporté dans leur étude avoir observé dans un tiers des cas (34\%) une alimentation complémentaire précoce, à partir de l'âge de 3 mois. L'alimentation complémentaire précoce est élevée en milieu urbain et la première contrainte est liée à une reprise de travail des mères $(80 \%)$. Les contraintes évoquées par les mères sont multiples où dans l'ensemble les principales causes sont la reprise de travail $(42 \%)$ et le manque de lait maternel $(42 \%)$. Ce résultat diffère de ceux de Azagoh-K. et al. (2013) et de Thimou et al. (2001) au Maroc qui a trouvé des proportions respectives de $24,1 \%$ et $9 \%$ pour la reprise du travail. Cette pratique d'alimentation complémentaire inadéquate observée en milieu urbain pourrait être due aux caractéristiques socio-économiques des mères où $80 \%$ ont une activité génératrice de revenu. De telles pratiques d'alimentation complémentaire encourent un double risque qui se traduit d'une part, par l'apparition des infections, de la diarrhée à l'éventuelle souillure des aliments de compléments mal élaborés et introduits (Kouassi et al., 2015). Njjumba et al. (2004) ont quant à eux trouvé que les raisons évoquées par les mères associés au milieu urbain. La consommation de céréales, racines, tubercules et de légumineuses-noix est associée au milieu rural (Figures 2 et 3 ).

étaient l'insuffisance du lait maternel, les activités pratiquées loin du domicile, les maladies maternelles, une augmentation de l'appétit du nourrisson. L'étude des déterminants d'une alimentation complémentaire au-delà de trois (03) mois a montré que le niveau d'instruction des mères, leurs activités, leurs origines et leurs connaissances en matière d'alimentation complémentaire sont les principaux facteurs qui influencent les pratiques d'alimentation complémentaire des nourrissons. II ressort une association entre la bouillie issue des farines infantiles vendues dans le commerce, la bouillie enrichie avec des matières protéiques et le milieu urbain alors que la bouillie locale est associée au milieu rural. Les bouillies issues des farines infantiles vendues dans le commerce et celles enrichies avec des matières protéiques sont fréquemment utilisées en milieu urbain (ThiombianoCoulibaly et al., 2004). Cette pratique d'alimentation observée en milieu urbain pourrait être due au niveau de revenu élevé de ces ménages et aux activités des mères. En milieu rural, $18 \%$ seulement des bouillies locales sont enrichies avec une matière protéique végétale "le soja". Cette pratique très précaire et inadéquate des mères en milieu rural est due à l'ignorance, aux manques d'informations sur les bonnes pratiques d'alimentation complémentaire et au bas niveau de revenu des ménages (Thomazic, 2003). En milieu rural, 64\% des bouillies locales sont produites exclusivement avec le maïs et la fréquence de consommation journalière de bouillie est faible. Ces résultats sont en accord avec ceux de Trèche, 2002 et de Kasole et al. (2013) qui ont trouvé au Congo Démocratique des bouillies locales exclusivement produite avec de la pâte fermentée de mais "le poto-poto". Njjumba et al. (2004) et Traore et al. (2005) ont aussi montré que le mais constitue l'aliment de base le plus fréquemment utilisé en milieu rural pour la confection des bouillies locales, la consommation journalière de bouillie est faible et on rencontre des bouillies de céréales peu enrichies avec des légumineuses locales (pâte d'arachide ou farine torrifiée de soja). La consommation de céréales, racines et tubercules, de produits carnés (viandes, poissons), de fruits et légumes, de produits laitiers (lait, yaourt, fromage), d'œufs sont associés au milieu urbain et le milieu rural est associé aux céréales, racines, tubercules et légumineuses-noix. L'alimentation des jeunes enfants est plus diversifiée en milieu urbain puisque les différents 
groupes d'aliments sont présents et accessibles. En milieu rural, les plats proposés aux enfants sont riches en composés glucidiques et sont moins accompagnés de protéines animales, des fruits, légumes et des produits laitiers. Ces résultats sont en accord avec ceux de Amoussa-Hounkpatin, (2011) et Azagoh-K. et al. (2013). Les principaux résultats obtenus au cours de cette étude sont basés essentiellement sur les déclarations des mères. Aussi, les pratiques d'alimentation

\section{CONCLUSION}

Cette étude met en exergue les insuffisances au niveau des pratiques d'alimentation des jeunes enfants âgés de 6 à 36 mois. Les bouillies locales produites en milieu rural sont peu enrichies avec des matières protéiques. Elles ont de faibles valeurs nutritionnelles, consommées complémentaire évoquées ne sauraient être considérées comme une évaluation de ces dernières. Cette étude constitue une description sommaire de l'alimentation complémentaire des enfants de 6 à 23 mois en particulier et de l'alimentation des jeunes enfants en général. Néanmoins, elle a l'avantage de faire l'état des lieux des bouillies consommées par les jeunes enfants de 6 à 36 mois et met en évidence l'importance de la bouillie dans l'alimentation des jeunes enfants au Sud-Bénin.

souvent une fois par jour et sont exclusivement préparées à base de maîs. La production de bouillies locales accessibles et adéquates pour couvrir les besoins nutritionnels et énergétiques des jeunes enfants doit être encouragée.

REMERCIEMENTS : Nous remercions les mères d'enfants âgés de 6 à 36 mois enquêtées dans les quatre départements ainsi que le Gouvernement Béninois pour le financement de cette étude.

\section{REFERENCES}

Amoussa-Hounkpatin W., 2011. Évaluation du potentiel de couverture des besoins en vitamines $\mathrm{A}$ des jeunes enfants à partir des sauces accompagnant les aliments de base consommés au Bénin. Thèse de Doctorat de l'Université Montpellier 2. p 1-109.

Ategbo E. A., 1993. Food and nutrition insecurity in northern Benin: impact on growth performance of children and on year to year nutritional status of adults. PhD Thesis Wageningen University, Netherlands. p 1-150.

Azagoh-K R, Enoh J, Niangue B, Cissé L, Oulai S, Andoh $\mathrm{J}, 2013$. Connaissances et pratiques des mères d'enfants de 6 à 18 mois relatives à la conduite du sevrage : cas de l'hôpital général de Marcory. Mali médical 2013 : Tome XXVIII, №4.

Black RE, Victora CG, Walker SP, Bhutta ZA, Christian $\mathrm{P}$, de Onis M, Ezzati M, Grantham-McGregor S, Katz J, Martorell R, Uauy R, 2013. Maternal and child undernutrition and overweight in lowincome and middle-income countries. Lancet 382 (9890) : 427-451.

Brown KH. \& Lutter CK, 2000. Potential of processed complementary food in the improvement of early childhood nutrition in Latina America. Nutrition bulletin, $n^{\circ} 21$.

Carbonel I., 1996. Amélioration des bouillies de sevrage préparées à partir d'aliments fermentés au Bénin. Mémoire pour l'obtention du DESS
Nutrition et Alimentation dans les pays en développement. Université Montpellier II. p 1-36.

Diagne-Guèye NR, Diack-Mbaye A, Dramé M, Diagne I, Fall AL, Camara B, Faye PM, Sylla A, Diouf S, Sy-signaté H, Sarr M, 2011. Connaisances et pratiques des mères sénégalaises vivant en milieu rural ou suburbain sur l'alimentation de leurs enfants, de la naissance à l'âge de six mois. Journal de pédiatrie et de puériculture : 24 , 161-166.

Dolan C, Mwangome M, Khara T, 2015. Extent of stunting and wasting in the same children. Panel in global nutrition report 2015: Actions and accountability to advance nutrition and sustainable development. International Food Policy Research Institute : 102-108.

Forouzanfar MH, Alexander L, Anderson HR, Bachman VF, Biryukov S, Brauer M, Burnett R, Casey D, Coates MM, Cohen A, Delwiche K, 2015. A systematic analysis for the global burden of disease study 2013. Lancet 386 (10010) : 22872323.

Global Nutrition Report (GNR) Partners, 2016. Global nutrition report from promise to impact ending malnutrition by 2030. p 7-20

INSAE, 2015. Enquête par grappes à indicateurs multiples (MICS) - rapport de résultats clésBénin. p 4-15

Kasole TL, Mulangu AM, Muganza AN, Ilunga PM, Wakam GK, Kitengewa FM, 2013. Allaitement 
maternel et diversification alimentaire à Lubumbashi (RDC) : besoin urgent d'éducation des mères pour le changement des habitudes. African Medical Journal : 8-12.

Kouassi AK, Adouko AE, Gnahe DA, Grodji GA, Kouakou BD, Gnakri D, 2015. Comparaison des caractéristiques nutritionnelles et rhéologiques des bouillies infantiles préparées par les techniques de germination et de fermentation. Int. J. Biol. Chem. Sci. 9(2) : 944-953.

Njjumba M. \& Ntozi PM, 2004. Mothers in the Informal Economy and Changes in Child Feeding and caring Roles in Kampala, Uganda. Africa Development : 114-130.

OMS/UNICEF, 2003. Recommandations relatives à l'allaitement maternel exclusif et l'alimentation complémentaire des jeunes enfants dans les pays en développement. OMS : Genève ; 130131.

OMS., 2012. Maternel, Infant, and Young Child Nutrition : Draft Comprehensive Implementation Plan. "Geneva. http://apps.who.int/gb/ebwha/pdf_files.

de Onis M, Dewey KG, Borghi E, Onyango AW, Blossner M, Daelmans B, Piwoz E, Branca F, 2013. The world health organization's global target for reducing childhood stunting by 2025 : Rationale and proposed actions. Maternal and child nutrition $9(2): 6-26$.

Salle B, 2009. Alimentation du nouveau-né et du nourrisson. Rapport 24 Février 2009. Tome 193, No 2, p 431-446.

Thiombiano-Coulibaly N, Rocquelin G, Eymard-Duvernay S, Zougmore ON, Traore SA, 2004. Effects of early extra fluid and food intake on breast milk consumption and infant nutritional status at 5 months of age in an urban and rural area of Burkina Faso. European Journal of Clinical Nutrition : 58, 80-89.

Thomazic M, 2003. Caractérisation de la commercialisation et de la vente de poto- poto. Mémoire de DESS, Université de Montpellier II. p 1-58

Thimou A, Mdaghri A, El Harim EM, Lamdouar BN, 2001. La diversification alimentaire d'après une enquête menée au centre de néonatalogie $d u$ CHU de Rabat. Médecine du Maghreb: 862001.

Traoré T, Vieu MC, Traoré SA, Trèche S, 2005. Effets de la durée de la période d'accoutumance sur les ingérés énergétiques à partir de bouillies de faible et de hautes densités énergétiques chez les nourrissons Burkinabè vivant en milieu naturel. Appetite : 2-5.

Trèche, S. 2002. Complementary foods in developing countries: importance, required characteristics, constraints and potential strategies for improvement. In Kolsteren P, Hoerée T, PerezCueto eds: Proceedings of the International Colloquium promoting growth and development of under-fives. Antwerpen : ITG Press : 132-148.

UNICEF, 2014. Rapport Final Enquête Qualitative au BENIN. Étude des déterminants des pratiques d'alimentation complémentaire des enfants de moins de 2 ans : cas de 3 localités au Bénin : Bounkoumbé, Djakotomey et Kétou. p 10-55.

Victora CG, Bahl R, Barros AJ, Franca GV, Horton S, Krasevec J, Murch S, Sankar MJ, Walker N, Rollins NC, 2016. Breastfeeding in the 21s Century: Epidemiology, Mechanisms, and Lifelong Effect. Lancet 387 (10017) : 475-490.

Zannou Tchoko J, Bouaffou KG, Kouame KG, Konan BA, 2011. Étude de la valeur nutritive de farines infantiles à base de manioc et de soja pour enfant en âge de sevrage. Bulletin de la Société Royale des Sciences de Liège $80: 748$ - 758. 